\title{
RELATO DE CASO
}

\section{ENTOMOFTOROMICOSE INTESTINAL: RELATO DE CASO}

\author{
Fábia Aparecida Carvalho, Jefferson Lessa S. de Macedo, \\ Jean Newton L. Costa e Mário A. P. Moraes
}

\begin{abstract}
Os autores relatam um caso de entomoftoromicose intestinal causada por Entomophthorales, em individuo de 19 anos, agricultor e sem doença associada. O paciente foi submetido a ressecção intestinal e o diagnóstico foi feito após análise da peça cirúrgica. Após revisão da literatura, são discutidos a evolução clínica, as características clinicopatológicas, as dificuldades no diagnóstico e o tratamento dessa entidade rara.
\end{abstract}

Palavras-chaves: Entomoftoromicose intestinal. Entomophthorales. Zigomicetos. Basidiobolus haptosporus.

As zigomicoses são doenças causadas por espécies das ordens Mucorales ${ }^{118} \mathrm{e}$ Entomophthorales ${ }^{2}{ }^{1015}$. Os fungos da ordem Entomophthorales são representados por Basidiobolus haptosporus (Basidiobolus ranarum), Entomophthora coronata (Conidiobolus coronatus) e Conidiobolus incongruus; o primeiro, causa infecção das regiões nasal e paranasal $^{315}$, enquanto as lesões causadas por Basidiobolus haptosporus estão localizadas no subcutâneo do tronco ou das extremidades e, eventualmente, no trato gastrintestinal como uma massa pseudotumoral ${ }^{25781622}$.

A presente descrição trata de um caso de entomoftoromicose intestinal provavelmente causado por Basidiobolus haptosporus.

\section{RELATO DE CASO}

Paciente de 19 anos, agricultor, natural e procedente de Araguaiana (Mato Grosso, Brasil), admitido no hospital com história de massa abdominal, evoluindo há 3 meses, associada à febre, sudorese, diarréia e perda de $9 \mathrm{~kg}$ no período. O paciente tinha antecedentes alimentares de ingestão de pães mofados.

Ao exame físico, estava em regular estado geral e à palpação do abdome havia uma tumoração na fossa ilíaca direita, de 4 por $6 \mathrm{~cm}$, endurecida, dolorosa e pouco móvel. Os achados laboratoriais revelaram leucocitose de

Serviço de Cirurgia Geral do Hospital das Forças Armadas e Departamento de Patologia da Faculdade de Ciências da Saúde da Universidade de Brasília, Brasília, DF.

Endereço para correspondência: Dr. Jefferson Lessa Soares de Macedo. SQN 313 Bloco ,J Apto. 203, 70766-100 Brasilia, DF

Recebido para publicação em 06/05/96.
$12.600 / \mathrm{mm}^{3}$, com $11 \%$ de eosinófilos, velocidade de hemossedimentação de $49 \mathrm{~mm} / \mathrm{h}$ e função hepática normal. O raio X do tórax era normal e no exame radiológico do trânsito intestinal observou-se grande lesão infiltrativa envolvendo válvula íleo-cecal, ceco e cólon ascendente com grande aumento das partes moles. À colonoscopia, visualizou-se, no cólon ascendente, mucosa edemaciada, hiperemiada, com segmentos ulcerados e pólipos de tamanho variados, sendo que essas alterações estendiam-se até o ceco. As biópsias realizadas tiveram laudo histopatológico sugestivo de doença de Crohn. Foi então instituído tratamento clínico com sulfasalazina ( $2 \mathrm{~g} / \mathrm{dia})$ e ácido fólico, durante 2 meses, sem melhora satisfatória.

Devido o paciente não apresentar melhora clínica e à possibilidade deste processo tumoral ter áreas de malignização, optou-se pelo tratamento cirúrgico. O paciente foi submetido à intervenção cirúrgica em 19/04/89, sendo encontrado tumor que iniciava no íleo terminal ( $5 \mathrm{~cm}$ distais) avançando pelo cólon ascendente até o ângulo hepático englobando válvula íleocecal, apêndice e ceco. O mesentério da região próxima ao tumor apresentava grandes linfonodos. Foi realizada hemicolectomia direita com ileocoloanastomose. O paciente evolui bem, recebeu alta no nono dia pós-operatório, sendo acompanhado ambulatorialmente.

No exame anatomopatológico, observou-se uma peça cirúrgica compreendendo íleo $(15 \mathrm{~cm})$, ceco, cólon ascendente e cólon transverso $(10 \mathrm{~cm})$. A serosa apresentava-se brilhante com múltiplas nodulaçôes superficiais, toda parede intestinal estava espessada com um máximo de $4,5 \mathrm{~cm}$ ao nível do íleo 
Relato de Caso. Carvalho FA, Macedo JLS, Costa JNL, Moraes MAP. Entomoftoromicose intestinal: relato de caso. Revista da Sociedade Brasileira de Medicina Tropical 30:65-68, jan-fev, 1997.

terminal. A mucosa intestinal nas áreas de espessamento apresentava-se ulcerada e hemorrágica sendo que o restante da mucosa mostrava-se muito edemaciada. À microscopia, observou-se que a mucosa e submucosa estavam congestas com poucos focos de infiltrado inflamatório. Nas camadas muscular e serosa, notava-se uma reação granulomatosa de células gigantes associadas a áreas de

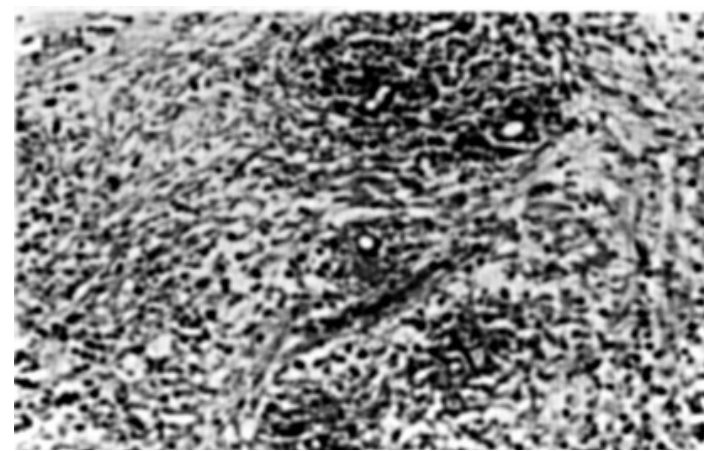

Figura 1 - Observam-se hifas vazias cortadas transversalmente cercadas pela substância eosinofilica do fenômeno de Splendore-Hoeppli (HE 200 x). necrose e células da inflamação aguda e crônica. As hifas largas e aparentemente vazias do fungo estavam presentes nas áreas de necrose, cercadas por uma substância eosinofílica, PAS positiva (fenômeno de Splendore-Hoeppli). Não havia invasão vascular ou trombose pelos fungos (Figuras 1 e 2). Não foi realizado isolamento do fungo por cultura.

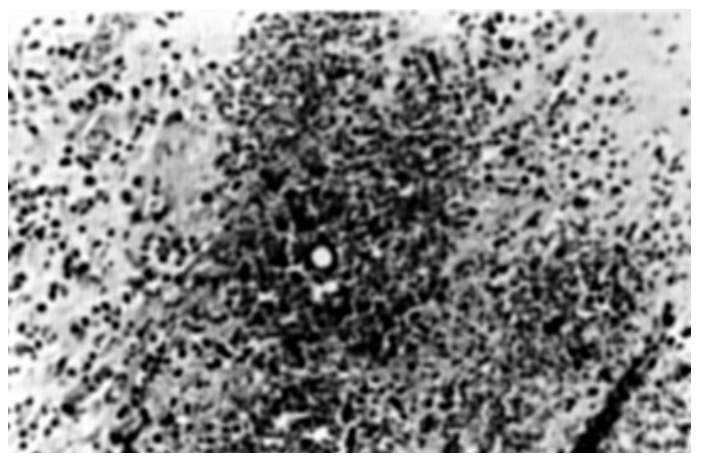

Figura 2 - Área de necrose envolvendo hifa cortada transversalmente associada ao fenômeno de SplendoreHoeppli (HE $200 x$ )

\section{DISCUSSÃO}

A infecção gastrintestinal devido a zigomicetos pode ser causada por espécies da ordem Mucorales 1217181920 ou da ordem Entomophthorales 2462223 . Entretanto, as características clínicas e histológicas das mucormicoses e das entomoftoromicoses são bem diferentes. A zigomicose intestinal por Mucorales acomete pacientes com uma doença de base (diabetes mellitus, leucemia, linfomas, desnutrição ou em uso de imunodepressores) $)^{1} \quad \begin{array}{llllll}11 & 12 & 18 & 19 & \text {, enquanto na }\end{array}$ infecção por Entomophthorales acometem pacientes a parentemente sadios ${ }^{2623}$. Nas seccões dos tecidos, a característica das hifas de Entomophthorales que mais chama a atenção é o material eosinofílico radiado que as envolve (Fenômeno de Splendore-Hoeppli); na reação tecidual predominam os eosinófilos com formação de granulomas ${ }^{24}$. A mais importante característica das hifas de Mucorales no estudo histopatológico é sua afinidade pelos vasos sangüíneos, que são invadidos e trombosados, levando a infartos ${ }^{18} 19$

O quadro clínico e as características histológicas da lesão deste paciente sugerem que o agente etiológico seja Basidiobolus haptosporus. No quadro clínico os dados favoráveis são o fato de o paciente não apresentar moléstias associadas e manifestar um acometimento intestinal isolado da doença. Quanto ao estudo histopatológico foram observadas as hifas do fungo envoltas por uma substância eosinofílica (fenômeno de SplendoreHoeppli) e ausência de invasão vascular pelo fungo.

O primeiro caso de infecção humana causada por Basidiobolus haptosporus foi reportado, em 1956, na Indonésia ${ }^{14}$. Desde então, casos têm sido reportados na Ásia, África e América 5791622 . A micose ocorre geralmente em crianças como uma massa endurecida, bem 
Relato de Caso. Carvalho FA, Macedo JLS, Costa JNL, Moraes MAP. Entomoftoromicose intestinal: relato de caso. Revista da Sociedade Brasileira de Medicina Tropical 30:65-68, jan-fev, 1997.

definida e envolvendo o tecido celular subcutâne $0^{578}$. O primeiro caso de entomoftoromicose intestinal causado por Basidiobolus haptosporus, com comprovação micológica, foi reportado em Brasília, $\mathrm{DF}^{2}$. As infecções gastrintestinais por zigomicetos podem levar muitas vezes à morte, principalmente quando são causadas por Mucorales, sendo que na maioria das vezes o diagnóstico só é feito à necrópsia ${ }^{12} 1920$. No presente caso foi possível fazer-se o diagnóstico em vida do paciente, com sucesso no tratamento.

O diagnóstico das entomoftoromicoses pode ser estabelecido por estudo histopatológico. As colorações especiais para fungos, como o PAS (ácido periódico-Schiff) e a impregnação pela prata (método de Grocott) ajudam na visualização do fungo, mas são dispensáveis pois a coloração por hematoxilina-eosina permite muito bem o reconhecimento das hifas $^{24} 25$.

O fenômeno de Splendore-Hoeppli fica mais evidente quando a lâmina é corada com hematina ácida de Baker. Esse fenômeno que caracteriza a entomoftoromicose consiste em um precipitado que cerca o fungo composto ao que se admite por complexos antígenoanticorpo e os eosinófilos têm uma presença marcante ${ }^{25}$. A ausência desse precipitado eosinofílico na mucormicose e a associação dessa infecção fúngica com outras condições debilitantes (diabetes, linfoma e leucemia) sugerem que a mucormicose seja uma infecção oportunística. Além disso, a mucormicose difere das outras infecções fúngicas oportunísticas porque tem como complicação clínicopatológica a trombose vascular ${ }^{18}$.

Para determinação do agente etiológico deve-se fazer cultura do material de biópsia gastrointestinal no meio de ágar Sabouraud com antibióticos. O crescimento dos fungos ocorre entre 2 a 5 dias de incubação ${ }^{58}$.

Neste caso não foi realizada cultura porque não houve suspeita no diagnóstico diferencial. Portanto, a chave do diagnóstico está centrado no contexto da distribuição geográfica da doença e possibilidade de ocorrência no local.

Alguns métodos sorológicos têm sido desenvolvidos para diagnóstico da zigomicose, como a imunofluorescência e a imunodifusão em gel, mas nenhum deles demonstrou utilidade real na prática clínica ${ }^{13}$.

O tratamento da entomoftoromicose intestinal não está definido, pois existem poucos casos em que o diagnóstico foi estabelecido "antemortem" ${ }^{621}$. Na maioria das vezes o diagnóstico só é estabelecido na necrópsia ${ }^{23}{ }^{26}$. Existe relato do uso com sucesso do iodeto de potássio ${ }^{4}$ e o tratamento cirúrgico com excisão da massa associado ao uso sistêmico de anfotericina $\mathrm{B}^{619}$. Interessante ressaltar que a entomoftoromicose intestinal pode ser tratada clinicamente com drogas antifúngicas, mas isto está na dependência de um diagnóstico precoce.

Desse modo por apresentar um quadro clínico inespecífico e ser uma entidade rara, a entomoftoromicose intestinal é subdiagnosticada, contribuindo para uma alta mortalidade e morbidade entre os pacientes por falta de diagnóstico.

\section{SUMMARY}

A case of intestinal entomophthoramycosis caused by Entomophthorales in a man with 19 years-old, farmer and without associated disease. The patient was submitted to a intestinal ressection and diagnosis was carried through after analisys of the surgical specimen. After a review of the literature, the clinical evolution, clinico-pathologic features, difficulties in diagnosis and treatment are discussed.

Key-words: Intestinal entomophthoramycosis. Entomophthorales. Zygomicetes. Basidiobolus haptosporus

\section{REFERÊNCIAS BIBLIOGRÁFICAS}

1. Agha FP. Mucormycosis of colon: early diagnosis and treatment.American Journal of Roentology 145:739, 1985.

2. Aguiar E, Moraes WC, Londero AT. Gastrointestinal entomophthoramycosis caused by Basidiobolus haptosporus. Mycopathologia 72:101-105, 1980.

3. Andrade ZA, Paula LA, Sherlock IA, Cheever AW. Nasal granuloma caused by Entomophtora coronata. The American Journal of Tropical Medicine and Hygiene 16:31-33, 1967.

4. Araújo RC,Agostini AA, Pereira NR, Magalhães AV, Raick AN. Ficomicose gstrica. In: Resumos do XII Congresso da Sociedade Brasileira de Patologia p. $72,1977$.

5. Bittencourt AL, Melo CR, Jalil OAM, Andrade ZA. Basidiobolomicose: apresentação de um caso. Revista do Instituto de Medicina Tropical de São Paulo 19:208-212, 1977. 
Relato de Caso. Carvalho FA, Macedo JLS, Costa JNL, Moraes MAP. Entomoftoromicose intestinal: relato de caso. Revista da Sociedade Brasileira de Medicina Tropical 30:65-68, jan-fev, 1997.

6. Bittencourt AL, Ayala MAR, Ramos EAG. A new form of abdominal zygomycosis different from mucormycosis: report of two cases and review of the literature. The American Journal of Tropical Medicine and Hygiene 28:564-569, 1979.

7. Bittencourt AL, Serra G, Sadigursky M, Araújo MGS, Campos MCS, Sampaio LCM. Subcutaneous zygomycosis caused by Basidiobolus haptosporus: presentation of case mimicking Burkitt's limphoma. The American Journal of Tropical Medicine and Hygiene 31:370-373, 1982.

8. Bittencourt AL, Arruda SM, Andrade JA, Carvalho EM. Basidiobolomycosis: a case report. Pediatric Dermatology 8:325-328, 1991.

9. Davis SR, Ellis DH, Goldwater P, Dimitriou S, Byard R. First human culture-proven australian case of entomophthoromycosis caused by Basidiobolus ranarum. Journal of Medical and Veterinary Mycology 32:225-230, 1994.

10. Eckert HL, Khoury GH, Pore RS, Gilbert EF, Gaskell JR. Deep Entomophtora phycomycotic infection reported for the first time in the United States. Chest 61:392-394, 1972.

11. Fingerote RJ, Seigel S,Atkinson MH, Lewkonia RM. Disseminated zygomycosis associated with lupus erythematosus. Journal of Rheumatology 17:1692-1694, 1990.

12. Gulati S, Barthakur G, Banerjee CK, Singhl S. Zygomycosis of colon. Indian Pediatrics 28:940$943,1991$.

13. Imwidthaya P, Srimuang S. Immunodiffusion test for diagnosing basidiobolomycosis. Mycopathologia 118:127-131, 1992.

14. Joe LF, Eng NIT, Pohan A, Muelen V, Emmons CW. Basidiobolus ranarum as a cause of subcutaneous mycosis in Indonesia. Archives of Dermatology 74:378-383, 1956.

15. King DS. Systematics of fungi causing entomophthoramycosis. Mycologia 71:731-745, 1979.
16. Koshi G, Kurien T, Sudarsanam D, Selvapandian A, Mammer KE. Subcutaneous phycomycosis caused by Basidiobolus: a report of three cases. Sabouraudia 10:237-243, 1972.

17. Laws on HH, Schamaman A. Gastric phycomycosis. British Journal of Surgery 61:743$746,1974$.

18. Lehrer RI, Howard DH, Sypherd PS, Edwards JE, Segal GP, Winston DJ. Mucormycosis: UCLA Conference. Annals of Internal Medicine 93:93108, 1980.

19. Lyon DT, Schubert TT, Mantia AG, Kaplan MH. Phycomycosis of the gastrointestinal tract. American Journal of Gastroenterology 72:379394, 1979.

20. Michalak DM, Cooney DR, Rhodes KH, Telander RL, Kleinberg F. Gastrointestinal mucormycosis in infants and children: a cause of gangrenous intestinal cellulitis and perforation. Journal of Pediatric Surgery 15:320-324, 1980.

21. Restrepo A.Treatment of tropical mycosis. Journal of American Academy of Dermatology 31:91-102, 1994.

22. Schmidt JH, Howard RJ, Chen JL, Pierson KK. First culture-proven gastrointestinal entomophthoromycosis in the United States: a case report and review of the literature. Mycopathologia 95:101-104, 1986.

23. Soares HL, Miranda D, Nunes A. Tropical phycomycosis involving the pelvic cavity and thighs in a Brazilian child. The American Journal of Tropical Medicine and Hygiene 23:701-703, 1974.

24. Williams AO. Pathology of phycomycosis due to Entomophthora and Basidiobolus species. Archives of Pathology 87:13-20, 1969.

25. Williams AO, Lichtenberg F, Smith JH, Martinson FD Ultrastructure of phycomycosis due to Entomophthora, Basidiobolus and associated " Splendore-Hoeppli" phenomenon. Archives of Pathology 87:459-468, 1969. 